Case Report

\title{
Ischemic Stroke in an HIV Positive Patient: An Initial Presentation of Neurosyphilis
}

\author{
Kalimullah Jan (D), ${ }^{1}$ Rebecca Hoe Hui Min, ${ }^{1}$ \\ Tan Seow Yen, ${ }^{2}$ and Shekhawat Ravindra Singh ${ }^{3,4}$ \\ ${ }^{1}$ Department of Medicine, Changi General Hospital, Singapore \\ ${ }^{2}$ Department of Infectious Diseases, Changi General Hospital, Singapore \\ ${ }^{3}$ Department of Neurology, Changi General Hospital, Singapore \\ ${ }^{4}$ National Neuroscience Institute, Singapore \\ Correspondence should be addressed to Kalimullah Jan; kohatian3659@yahoo.com
}

Received 29 September 2017; Accepted 21 January 2018; Published 13 February 2018

Academic Editor: Dominic B. Fee

Copyright (C) 2018 Kalimullah Jan et al. This is an open access article distributed under the Creative Commons Attribution License, which permits unrestricted use, distribution, and reproduction in any medium, provided the original work is properly cited.

\begin{abstract}
Ischemic stroke occurring in patients with human immunodeficiency virus (HIV) needs to be approached with a vast differential diagnosis in mind. We report a case of middle-aged male patient with immune reconstituted HIV on therapy without known cardiovascular risk factors who had a right middle cerebral artery territory infarct. After a thorough evaluation, he received a final diagnosis of neurosyphilis-associated vasculitis leading to stroke. He recovered without any neurological deficits following treatment with intravenous benzylpenicillin. Neurosyphilis is an easily diagnosed and treatable cause of a stroke that can be an initial presentation of neurosyphilis but requires a high index of suspicion.
\end{abstract}

\section{Case Report}

A 57-year-old Chinese male presented to the Emergency Department (ED) of a tertiary hospital with complaints of transient left upper and lower limb weakness associated with a left facial droop and slurring of speech that had lasted for half an hour. He denied any history of similar events. He had a known history of human immunodeficiency virus (HIV) infection on highly active antiretroviral therapy (HAART) for the past four years. He was a nonsmoker. There was no family history of stroke.

On initial presentation, he was hemodynamically stable with blood pressure of 165/98 mmHg. Physical examination revealed dysarthria with a left facial droop and left-sided pronator drift. The rest of the examination was unremarkable. His capillary blood glucose was normal. His National Institutes of Health Stroke Scale (NIHSS) score on arrival to ED was 3 points.

Urgent noncontrast computed tomography (CT) of the brain was obtained in ED within minutes of arrival. This showed acute right middle cerebral artery (MCA) territorial infarct without haemorrhagic conversion. There was presence of mild right frontoparietal mass effect. His ASPECTS (Alberta Stroke Programme Early CT Score) was 5. In view of rapidly resolving symptoms and low NIHSS score, thrombolysis with recombinant Tissue Plasminogen Activator was not offered.

He was admitted to the acute stroke unit after starting aspirin (100 mg) and Atorvastatin (40 mg). For his HIV, he was being prescribed daily doses of abacavir, efavirenz, and lamivudine, to which he claimed compliance. His last CD4 count was 533 cells/uL (lab range: 280-1430) one year ago. The most recent HIV viral load performed a week before admission was detectable but less than 20 copies $/ \mathrm{mL}$.

His initial investigations revealed a normal electrocardiogram, troponin, random glucose, urea, electrolytes, liver function test, coagulation profile, and full blood count. Fasting low density lipoprotein cholesterol (LDL-c) was $2.28 \mathrm{mmol}$ and his HbAlc was less than $4.3 \%$. His subsequent inpatient blood pressure readings were normal and antihypertensive agents were not required. His chest radiograph, transthoracic echocardiography, Doppler ultrasound of bilateral carotids, and 24-hour Holter monitoring were normal. 
TABLE 1: Serum thrombophilia and autoimmune tests.

Test
Serum Venereal Disease Research Laboratory (VDRL
Serum Treponema pallidum particle agglutination
(TPPA) test
Anti-cardiolipin IgM antibody
Anti-cardiolipin IgG antibody
Anti-thrombin III
Protein C
Protein S (functional)
Factor V Leiden mutation
Anti-nuclear antibodies (ANA)
Anti-double stranded DNA
Anti-neutrophil cytoplasmic antibodies (ANCA)
(anti-myeloperoxidase, anti-proteinase 3)
Extracted nuclear antigen (ENA) profile

${ }^{1} \mathrm{MPL}=1 \mathrm{microgram} / \mathrm{ml} \mathrm{IgM},{ }^{2} \mathrm{GPL}=1 \mathrm{microgram} / \mathrm{ml} \mathrm{IgG},{ }^{3}$ anti-Sjögren's syndrome-related antigen A, ${ }^{4}$ anti-Sjögren syndrome type B antigen, ${ }^{5}$ antitopoisomerase I, and ${ }^{6}$ anti-histidyl tRNA synthetase.
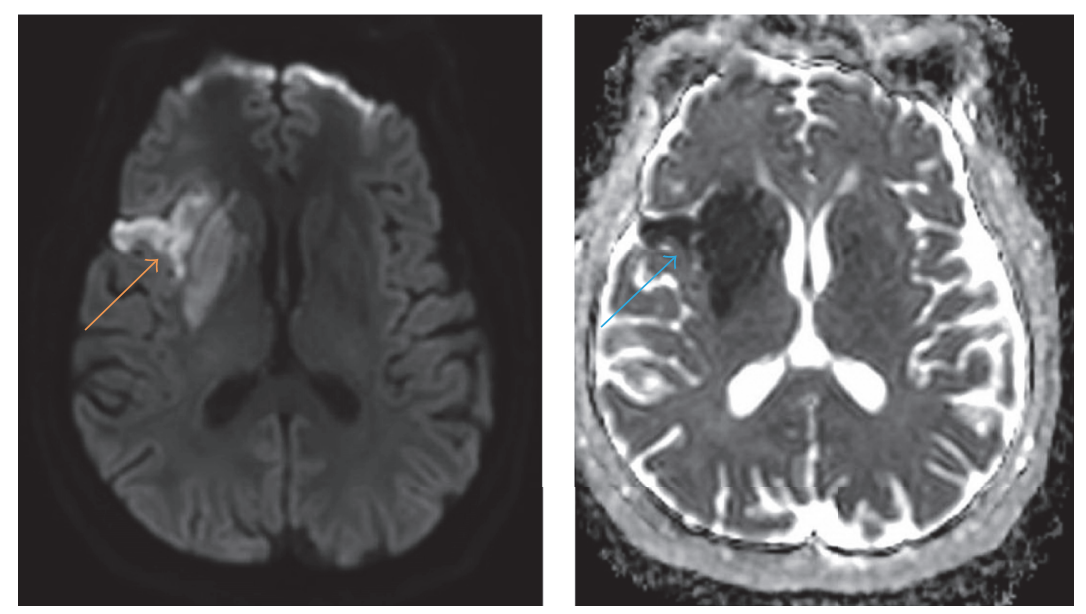

FIGURE 1: MRI brain reveals an area of diffusion restriction over the right MCA territory (orange arrow) with corresponding signal loss on Apparent Diffusion Coefficient (ADC) mapping (blue arrow), suggesting acute ischemic infarct.

Magnetic resonance imaging (MRI) of the brain was performed while he was an inpatient, revealing a right MCA territory infarct (Figure 1). Time of flight magnetic resonance angiography (ToF-MRA) showed suggestion of mild stenosis of the middistal right M1 segment of the MCA with no flow limitation.

In view of the absence of known cardiovascular risk factors, a thrombophilia and autoimmune workup was performed as shown in Table 1.

CT angiography of the circle of Willis was performed to look for evidence of vasculitic phenomena. As shown in Figure 2, there was bilateral moderate luminal irregularity in the M2 branches of the MCAs (more pronounced on the right, as shown by arrows) with less pronounced changes in the A2 segments of the anterior cerebral arteries and their branch vessels, raising suspicion for small to medium vessel vasculitis.

A lumbar puncture was performed, which showed 203 white blood cells (WBC) with lymphocytes accounting for 90\%. His cerebrospinal fluid (CSF) protein was raised at $0.81 \mathrm{~g} / \mathrm{L}(0.10-0.40 \mathrm{~g} / \mathrm{L})$. CSF VDRL was positive at titres of $1: 8$ and his CSF Syphilis LIA-IgG was positive as well, thus confirming neurosyphilis.

He received a diagnosis of ischemic stroke secondary to neurosyphilitic vasculitis. The infectious diseases team was consulted and he was started on intravenous benzylpenicillin 


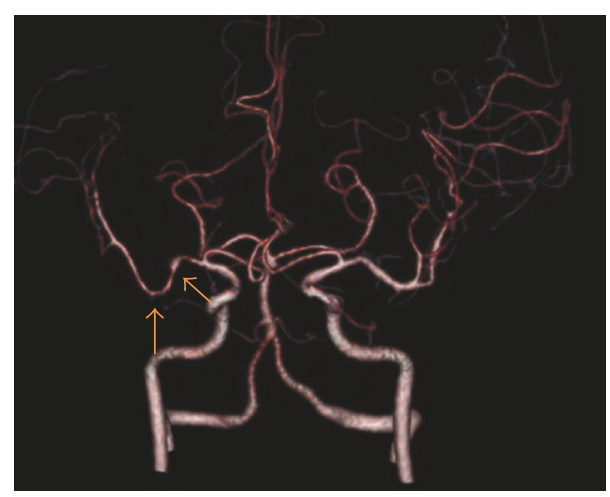

FIGURE 2: CT angiogram of the circle of Willis showing multiple areas of luminal irregularity.

4 million units 4 hourly for a total of 14 days. His aspirin and atorvastatin were discontinued. He recovered without any neurological sequelae during the course of his admission.

\section{Discussion}

Cerebrovascular disease is common in patients with HIV, occurring in up to $1.9 \%$ of HIV-infected patients [1]. In particular, patients with the acquired immunodeficiency syndrome (AIDS) have been reported to be at increased risk of both ischemic and haemorrhagic strokes, of which ischemic stroke is more frequent [2]. The pathogenesis of ischemic stroke in HIV is not well understood, with multiple postulated mechanisms including cardioembolism, coagulopathy, opportunistic infections, and HAART-related accelerated atherosclerosis, as well as primary vasculitis of the cerebral nervous system [3-5].

Patients with HIV are also susceptible to the atherosclerotic mechanisms affecting the majority of patients with stroke, and evaluation of stroke in HIV patients should similarly include Doppler ultrasound of the carotid vessels and cardiac evaluation. These investigations are useful to identify other conditions associated with HIV that predispose to stroke, including accelerated atherosclerosis, endocarditis, or cardiovascular complications of HIV infection [6].

MRA performed with MRI of the brain may reveal evidence of vasculitis in patients with HIV. Vasculitis in HIV is generally secondary to opportunistic infections or lymphoproliferative diseases [7]. In patients in whom the above have been excluded, HIV-related vasculitis is one consideration. This has been reported to occur in up to $20 \%$ of patients with HIV [8]. While the pathophysiology behind HIV-related vasculitis is not well defined, this condition is more commonly described in patients with low CD4 counts. HIV-related vasculitis can involve both intra- and extracranial arteries. HIV-associated intracranial vasculitis has been described as fusiform aneurysms involving the major arteries of the circle of Willis and the second- and third-order branches [9]. Tipping et al. described an association between intracranial vasculitis and low CD4 counts, while extracranial vasculitis appeared to be more common in patients with preserved CD4 counts [8]. This could reflect underlying differences in their pathogenesis.

As sexually transmitted diseases, syphilis and HIV frequently coexist. Amongst patients with HIV, those with lower CD4 counts are more likely to have concomitant neurosyphilis [10]. Central nervous system involvement is an uncommon manifestation of syphilis, occurring in less than $10 \%$ of patients with syphilis [11]. A study of HIV-infected patients by Ghanem et al. identified CD4 count of less than $350 \mathrm{cells} / \mathrm{ml}$ and lack of HAART treatment as predictors of neurosyphilis [12]. Lower CD4 counts have also been found to be associated with an increased risk of ischemic stroke $[4,7,13]$. Neurosyphilis can manifest as meningovascular syphilis, including meningitis or vasculitis, or parenchymal involvement in the form of general paresis or tabes dorsalis [14]. Meningovascular syphilis refers to the occurrence of an acute focal neurological deficit in a vascular territory of the brain or spinal cord based on clinical and/or imaging findings. Meningovascular syphilis has been reported to account for $24-53 \%$ of neurosyphilis, with a reduction in the proportion of late stages of neurosyphilis in the antibiotic era [15]. The mechanisms described include small vessel arteritis, also known as Nissl-Alzheimer arteritis, medium to large vessel arteritis, also known as Heubner arteritis, and less commonly extrinsic compression from aortic aneurysms $[16,17]$. Angiographically, meningovascular syphilis tends to display focal segmental narrowing and dilatation, resulting in the appearance of "beading" [18]. However, these findings are nonspecific and can resemble findings in other medium and large vessel vasculitides, and an underlying HIV-associated vasculopathy cannot be excluded.

Serologic screening for syphilis should be performed in patients with cryptogenic stroke, particularly in those with risk factors for syphilis $[14,19]$. The presence of a positive treponemal test should prompt further evaluation for neurosyphilis in the form of a lumbar puncture [20]. CSF features suggestive of neurosyphilis include lymphocytic pleocytosis, low glucose, and raised protein [14], which may occur in HIV alone as well [21]. Positive CSF VDRL is diagnostic of neurosyphilis, although the absence of CSF VDRL does not exclude it [22]. In patients with high suspicion for neurosyphilis, further evaluation in the form of CSF FTAABS is recommended [22].

Treatment of neurosyphilis is the same in HIV positive and negative patients and involves a 10- to 14-day course of high-dose intravenous benzylpenicillin [22]. Repeat CSF evaluation every 6 months is recommended in patients whose pretreatment CSF showed pleocytosis until the CSF cell count normalizes. Declining nontreponemal titres may be predictive of normalization of CSF white cell count [23]. Neurological deficits frequently but not always resolve following treatment. Conventional therapy including antiplatelets and statins for secondary prevention of stroke has not been well studied in patients with HIV or neurosyphilis. However, it is reasonable to suggest that such therapy be implemented in the absence of contraindications, bearing in mind the potential for drug interactions with HAART $[6,24,25]$. With the increasing life expectancy of patients with HIV, the incidence of stroke in patients with HIV may be expected to rise. As 
such, there may be a role for further investigation into the management of stroke in patients with HIV.

In our patient, despite his age, the absence of cardiovascular risk factors as well as his known diagnosis of retroviral disease prompted further evaluation which included not just screening for syphilis but also for other prothrombotic states. His positive serum VDRL and TPPA serologies in the absence of clinical features of syphilis confirmed the diagnosis of latent syphilis. Further history from the patient was unrevealing for any recent sexual encounters or positive contact history. His subsequent positive CSF VDRL confirms the diagnosis of neurosyphilis, while his cerebrovascular imaging was consistent with that of vasculitis, leading to the diagnosis of ischemic stroke secondary to meningovascular syphilis. As this patient had no other cardiovascular risk factors, and his LDL-c was normal, the decision was made to discontinue his aspirin and statin. Our patient's HIV infection was well suppressed with HAART. This suggests that screening for neurosyphilis in HIV patients who present with ischemic stroke is essential regardless of the degree of immunosuppression.

\section{Conclusion}

In summary, patients with HIV are predisposed to cerebrovascular diseases through a variety of aetiologies, of which neurosyphilis is an easily diagnosed and treatable cause. While these mechanisms of stroke are commoner in patients with AIDS, in the absence of identifiable risk factors, care should be taken to exclude an underlying cause in order to ensure correct treatment and prevention of recurrent episodes of debilitating stroke. Also, it is to be noted that stroke can be an initial presentation of neurosyphilis and requires a high index of suspicion.

\section{Disclosure}

Kalimullah Jan (formerly) worked at the Department of Neurosurgery, Aga University Hospital, Karachi, Pakistan.

\section{Conflicts of Interest}

The authors declare that they have no conflicts of interest.

\section{Authors' Contributions}

Kalimullah Jan and Rebecca Hoe Hui Min contributed equally to this manuscript and share first authorship.

\section{References}

[1] S. Evers, D. Nabavi, A. Rahmann, C. Heese, D. Reichelt, and I. W. Husstedt, "Ischaemic Cerebrovascular Events in HIV Infection," Cerebrovascular Disease, vol. 15, pp. 199-205, 2003.

[2] J. W. Cole, A. N. Pinto, J. R. Hebel et al., "Acquired Immunodeficiency Syndrome and the Risk of Stroke," Stroke, vol. 35, no. 1, pp. 51-56, 2004.

[3] L. A. Benjamin, A. Bryer, S. Lucas, A. Stanley, T. J. Allain, E. Joekes et al., "Arterial ischemic stroke in HIV: defining and classifying etiology for research studies," Neurology - Neuroimmunology Neuroinflammation, vol. 3, no. 1, p. e254, 2016.
[4] S. Sen, A. A. Rabinstein, M. S. Elkind, and W. J. Powers, "Recent developments regarding human immunodeficiency virus infection and stroke," Cerebrovascular Diseases, vol. 33, no. 3, pp. 209-218, 2012.

[5] G. Melica, P. Brugieres, A.-S. Lascaux, Y. Levy, and J.-D. Lelièvre, "Primary vasculitis of the central nervous system in patients infected with HIV-1 in the HAART era," Journal of Medical Virology, vol. 81, no. 4, pp. 578-581, 2009.

[6] L. A. Benjamin, A. Bryer, H. C. A. Emsley, S. Khoo, T. Solomon, and M. D. Connor, "HIV infection and stroke: current perspectives and future directions," The Lancet Neurology, vol. 11, no. 10, pp. 878-890, 2012.

[7] I. Corral, C. Quereda, A. Moreno et al., "Cerebrovascular ischemic events in HIV-1-infected patients receiving highly active antiretroviral therapy: incidence and risk factors," Cerebrovascular Disease, vol. 27, no. 6, pp. 559-563, 2009.

[8] B. Tipping, L. De Villiers, H. Wainwright, S. Candy, and A. Bryer, "Stroke in patients with human immunodeficiency virus infection," Journal of Neurology, Neurosurgery \& Psychiatry, vol. 78, no. 12, pp. 1320-1324, 2007.

[9] A. A. Khalek, A. Razek, H. Alvarez, S. Bagg, S. Refaat, and M. Castillo, "Imaging spectrum of CNS vasculitis," RadioGraphics, vol. 34, no. 4, pp. 873-894, 2014.

[10] C. M. Marra, C. L. Maxwell, S. L. Smith et al., "Cerebrospinal fluid abnormalities in patients with syphilis: association with clinical and laboratory features," The Journal of Infectious Diseases, vol. 189, no. 3, pp. 369-376, 2004.

[11] T. M. Abkur, G. S. Ahmed, N. O. Alfaki, and M. O'Connor, "Neurosyphilis presenting with a stroke-like syndrome," BMJ Case Reports, 2015.

[12] K. G. Ghanem, R. D. Moore, A. M. Rompalo, E. J. Erbelding, J. M. Zenilman, and K. A. Gebo, "Neurosyphilis in a clinical cohort of HIV-1-infected patients," AIDS, vol. 22, no. 10, pp. 1145-1151, 2008.

[13] F. C. Chow, P. Bacchetti, A. S. Kim, R. W. Price, and P. Y. Hsue, "Effect of CD4+ cell count and viral suppression on risk of ischemic stroke in HIV infection," AIDS, vol. 28, no. 17, pp. 2573-2577, 2014

[14] L. M. Chahine, R. N. Khoriaty, W. J. Tomford, and M. S. Hussain, "The changing face of neurosyphilis," International Journal of Stroke, vol. 6, no. 2, pp. 136-143, 2011.

[15] M. Á. Conde-Sendín, R. Amela-Peris, Y. Aladro-Benito, and A. Angel-Moreno Maroto, "Current clinical spectrum of neurosyphilis in immunocompetent patients," European Neurology, vol. 52, no. 1, pp. 29-35, 2004.

[16] J. Bäuerle, A. Zitzmann, K. Egger, S. Meckel, C. Weiller, and A. Harloff, "The great imitator-still today! A case of meningovascular syphilis affecting the posterior circulation," Journal of Stroke and Cerebrovascular Diseases, vol. 24, no. 1, pp. el-e3, 2015.

[17] H. Nakane, Y. Okada, S. Ibayashi, S. Sadoshima, and M. Fujishima, "Brain infarction caused by syphilitic aortic aneurysm: a case report," Angiology, vol. 47, no. 9, pp. 911-917, 1996.

[18] T. C. Brightbill, I. H. Ihmeidan, M. J. D. Post, J. R. Berger, and D. A. Katz, "Neurosyphilis in HIV-positive and HIVnegative patients: neuroimaging findings," American Journal of Neuroradiology, vol. 16, no. 4, pp. 703-711, 1995.

[19] D. J. Cordato, S. Djekic, S. R. Taneja et al., "Prevalence of positive syphilis serology and meningovascular neurosyphilis in patients admitted with stroke and TIA from a culturally diverse 
population (2005-09)," Journal of Clinical Neuroscience, vol. 20, no. 7, pp. 943-947, 2013.

[20] C. A. Rodgers and S. Murphy, "Diagnosis of neurosyphilis: appraisal of clinical caseload," Genitourinary Medicine, vol. 73, no. 6, pp. 528-532, 1997.

[21] D. W. Marshall, R. L. Brey, W. T. Cahill, R. W. Houk, R. A. Zajac, and R. N. Boswell, "Spectrum of cerebrospinal fluid findings in various stages of human immunodeficiency virus infection," JAMA Neurology, vol. 45, no. 9, pp. 954-958, 1988.

[22] K. A. Workowski and S. M. Berman, "Centers for Disease Control and Prevention, Sexually transmitted diseases treatment guidelines," MMWR Recommendations and Reports, vol. 64, no. 3, pp. 1-137, 2015.

[23] C. M. Marra, C. L. Maxwell, L. C. Tantalo, S. K. Sahi, and S. A. Lukehart, "Normalization of serum rapid plasma reagin titer predicts normalization of cerebrospinal fluid and clinical abnormalities after treatment of neurosyphilis," Clinical Infectious Diseases, vol. 47, no. 7, pp. 893-899, 2008.

[24] E. J. Singer, M. Valdes-Sueiras, D. L. Commins, W. Yong, and M. Carlson, "HIV stroke risk: evidence and implications," Therapeutic Advances in Chronic Disease, vol. 4, no. 2, pp. 61-70, 2013.

[25] R. Behrouz, A. R. Malek, and R. I. Chichkova, "Meningovascular syphilis: revisiting an old adversary," Practical Neurology, pp. 32-37, 2011. 


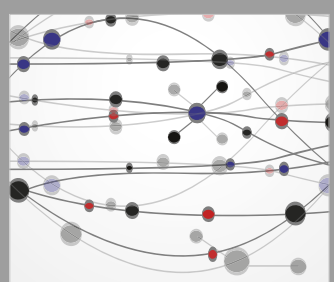

The Scientific World Journal
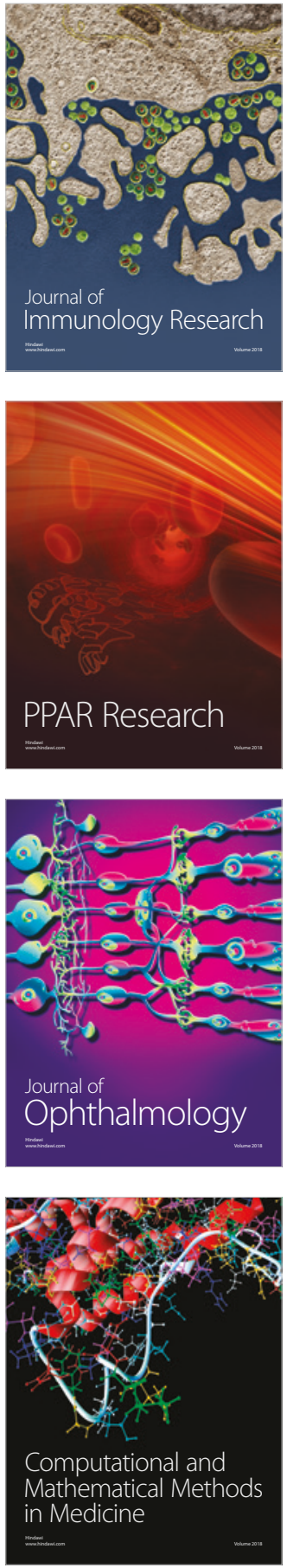

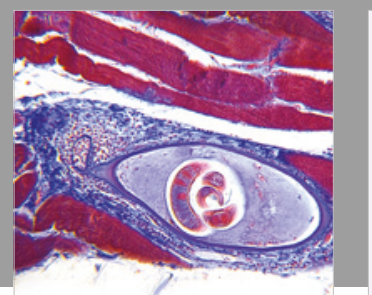

Gastroenterology Research and Practice

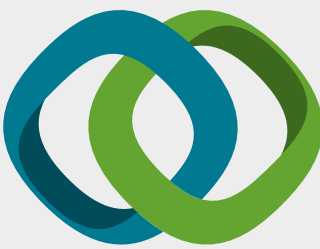

\section{Hindawi}

Submit your manuscripts at

www.hindawi.com
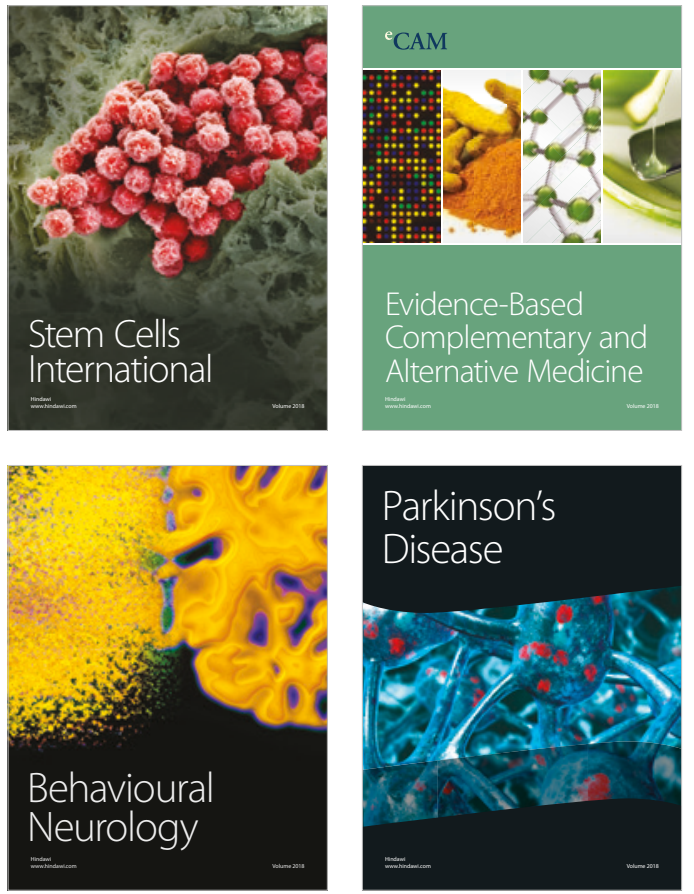

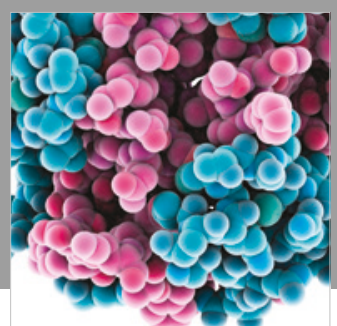

ournal of

Diabetes Research

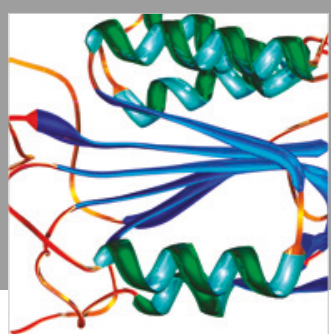

Disease Markers
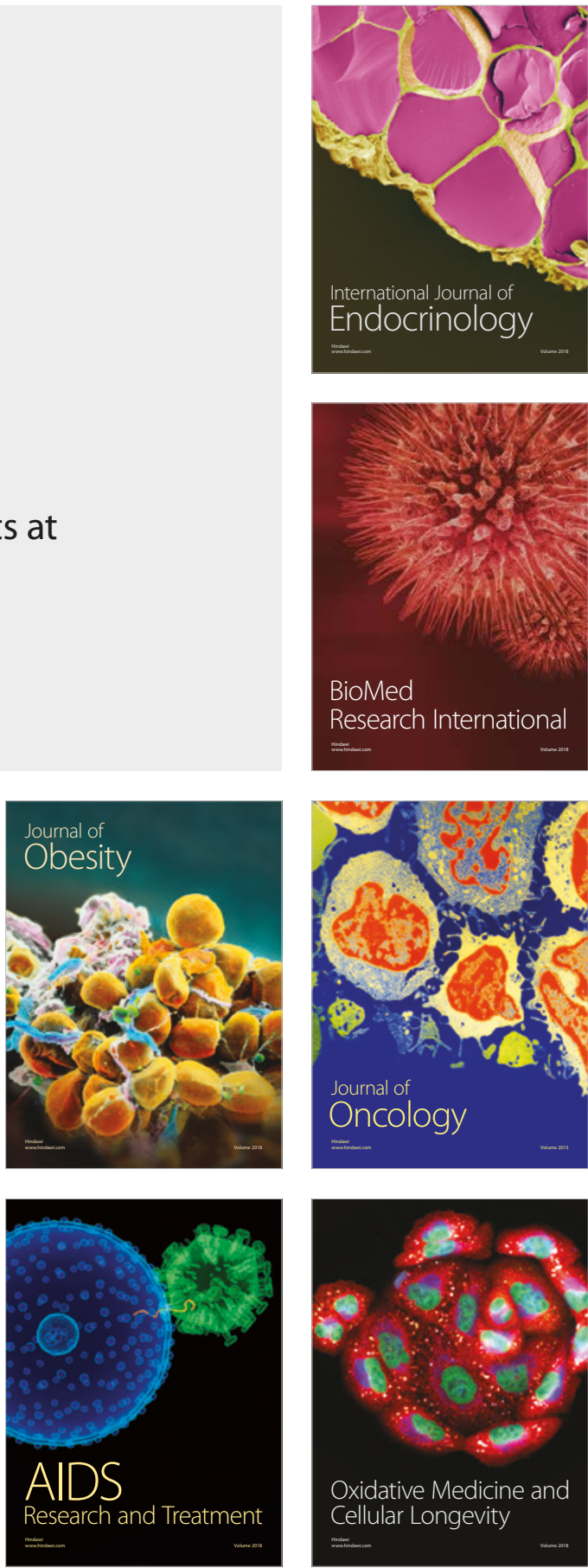